\title{
Curvature-Induced Defect Unbinding in Toroidal Geometries
}

\author{
Mark Bowick, ${ }^{1}$ David R. Nelson, ${ }^{2}$ and Alex Travesset ${ }^{3}$ \\ 1 Physics Department, Syracuse University, \\ Syracuse NY 13244-1130, USA \\ 2 Lyman Laboratory of Physics, Harvard University, \\ Cambridge MA 02138, USA \\ and \\ 3 Physics Department, Iowa State and Ames Natl. Lab., \\ Ames IA 50011-3160, USA
}

\begin{abstract}
Toroidal templates such as vesicles with hexatic bond orientational order are discussed. The total energy including disclination charges is explicitly computed for hexatic order embedded in a toroidal geometry. Related results apply for tilt or nematic order on the torus in the one Frank constant approximation. Although there is no topological necessity for defects in the ground state, we find that excess disclination defects are nevertheless energetically favored for fat torii or moderate vesicle sizes. Some experimental consequences are discussed.

PACS numbers: PACS numbers: 64.60Fr, 05.40.+j, 82.65.Dp
\end{abstract}

\section{INTRODUCTION}

Amphiphilic molecules in water or oil solutions have been intensely investigated over the last decade in a variety of settings. Amphiphiles in aqueous solution, under appropriate conditions, have been experimentally observed to form vesicles with the topology of the sphere, torus [1] and even higher genus surfaces [2]. Other experimental studies have focused on the phases of amphiphilic films as a function of temperature. It is by now well established that the high temperature fluid phase goes into a smectic-C $L_{\beta^{\prime}}$ phase through an intermediate rippled $P_{\beta^{\prime}}$ phase. Within the $L_{\beta^{\prime}}$ phase itself there are several other phases characterized by the degree of tilt and hexatic bond order [3, 4]. This beautiful experimental work may provide insight into biological problems such as membrane fusion [5], where it has been argued [6, 7] that molecular tilt plays an important role.

A remarkable understanding of the shapes of fluid amphiphilic systems has been provided by physical methods based on the Helfrich Hamiltonian 8] and its variations [9, 10]. In [1], the problem of fluctuating smectic$\mathrm{C}$ membranes, previously investigated in [17] for planar films, was addressed and predictions for the shape as a function of the elastic constants were presented. It was found that toroidal vesicles were favored for some parameters. It was assumed, however, that free disclinations are energetically unfavorable and may therefore be ignored unless, as in the case of the sphere, topological constraints require them.

The main result of this paper is that disclinations can be energetically favored over a wide range of parameters, even when not required by topological constraints. We treat vesicles that are topologically torii (closed surfaces of genus one). The Gauss-Bonnet theorem for torii requires a vanishing total disclination charge. We assume hexatic order in the tangent plane of the torus, arising from an anisotropic liquid phase of molecules with zero shear modulus. If $\left(x_{1}, x_{2}\right)$ are coordinates on the torus, specified in three dimensions by a function $\vec{R}\left(x_{1}, x_{2}\right)$, local hexatic order can be described by a bond angle field $\theta\left(x_{1}, x_{2}\right)$ (up to rotations by $2 \pi / 6=60^{\circ}$ ) defined relative to the local tangent vectors $\overrightarrow{e_{1}} \propto \partial_{1} \vec{R}$ and $\overrightarrow{e_{2}} \propto \partial_{2} \vec{R}$. On the torus, this pair can be chosen to be nonsingular and orthonormal everywhere; $\overrightarrow{e_{i}} \cdot \overrightarrow{e_{j}}=\delta_{i j}$. As discussed, e.g., by David [18], the usual hexatic energy on such a curved surface can be written

$$
E=\frac{1}{2} K_{A} \int d^{2} x \sqrt{g}\left(\partial_{i} \theta-\Omega_{i}\right)\left(\partial_{j} \theta-\Omega_{j}\right) g^{i j}
$$

where $K_{A}$ is the hexatic stiffness constant, $g^{i j}\left(x_{1}, x_{2}\right)$ is the inverse of the metric tensor

$$
g_{i j}=\frac{\partial \vec{R}}{\partial x_{i}} \cdot \frac{\partial \vec{R}}{\partial x_{j}}
$$

and $g=\operatorname{det} g_{i j}$.

The vector-potential-like function $\Omega_{j}\left(x_{1}, x_{2}\right)$ in Eq.(1) describes the geometric frustration which arises when vector or tensor order parameters are parallel transported on curved surfaces. This "spin connection" can be computed from covariant derivatives acting on $\overrightarrow{e_{1}}$ and $\overrightarrow{e_{2}}$; the "curl" of $\Omega$ (when appropriately defined on a curved surface) is proportional to the local Gaussian curvature [18].

Disclinations can be inserted into the free energy (11) just as in flat space. If $N$ defects with charges $q_{j}= \pm 1$ are present on the torus, we first define a defect density $s$ as a function of $x \equiv\left(x_{1}, x_{2}\right)$, namely

$$
s(x)=\frac{2 \pi}{6} \sum_{j=1}^{N} q_{j} \delta^{(2)}\left(x-x_{j}\right) / \sqrt{g\left(x_{j}\right)} .
$$

As discussed, e.g., in [18, 19, 20], minimizing (11) sub- 
ject to a fixed arrangement of $N$ defects, leads to

$$
\begin{aligned}
E & =\frac{K_{A}}{2} \int d^{2} x \sqrt{g(x)} \int d^{2} y \sqrt{g(y)} \\
& \times[s(x)-K(x)] G(x, y)[s(y)-K(y)] \\
& +\frac{\kappa}{2} \int d^{2} x \sqrt{g(x)} H^{2}(x)
\end{aligned}
$$

where $K(x)$ is the Gaussian curvature and $G(x, y)$ is the inverse Laplacian on the torus. Up to subtractions which eliminate "zero modes" (see Sec. III), $G(x, y)$ obeys

$$
\begin{aligned}
\nabla_{x}^{2} G(x, y) & =\frac{1}{\sqrt{g}} \partial_{i}\left\{\sqrt{g} g^{i j} \partial_{j}\right\} G(x, y) \\
& =\delta^{(2)}(x-y) / \sqrt{g(x)}
\end{aligned}
$$

The first term of (4) arises directly from (11) and represents a kind of two-dimensional electrostatics in curved space. As discussed below, this electrostatics can lead to a lower energy when positive and negative disclinations are placed at positions on the surface which approximately match the local Gaussian curvature. To account for the bending energy of the surface, we have added the second (Helfrich) term, where the bending rigidity coupling is $\kappa \approx(1-10) \mathrm{k}_{B} T$ for lipid bilayers and $H(x)$ is the mean curvature. Defect core energies, which depend on short distance physics not accounted for in this continuum approach, will be added later.

Although a representation of the physics of geometrical frustration like (11) is possible locally on any smooth surface, coordinates for genus one surfaces (the torus) can be found which admit such a representation globally. One such coordinate system is shown in Fig 1 where a point on the torus is specified by

$$
\vec{R}(\alpha, \theta)=\left(\begin{array}{c}
{\left[R_{1}+R_{2} \cos \alpha\right] \cos \theta} \\
{\left[R_{1}+R_{2} \cos \alpha\right] \sin \theta} \\
R_{2} \sin \alpha
\end{array}\right)
$$

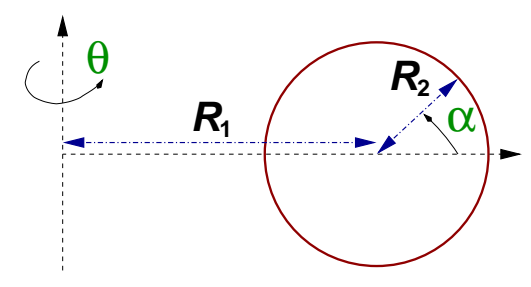

FIG. 1: Coordinates $(\alpha, \theta)$ defining the torus.

Here $R_{2}<R_{1}$ so that the torus does not intersect itself. The Gaussian curvature associated with (6) is a function of $\alpha$ only,

$$
K(\alpha)=\frac{\cos \alpha}{R_{1} R_{2}\left[1+\frac{R_{2}}{R_{1}} \cos \alpha\right]}
$$

Note that $K$ is positive on the outside wall of the torus $\left(-\frac{\pi}{2}<\alpha<\frac{\pi}{2}\right)$ and negative on the inside wall $\left(\frac{\pi}{2}<\alpha<\frac{3 \pi}{2}\right)$. Although the $(\alpha, \theta)$ coordinate system has a clear physical interpretation, most of our results are obtained using the conformal coordinates introduced in the Appendix [21]. Upon replacing $\alpha$ by a new angular variable $\varphi(0 \leq \varphi \leq 2 \pi)$ defined by

$$
\cos \varphi=\frac{R_{1} \cos \alpha+R_{2}}{R_{1}+R_{2} \cos \alpha},
$$

one obtains a locally flat metric, which greatly simplifies the calculations.

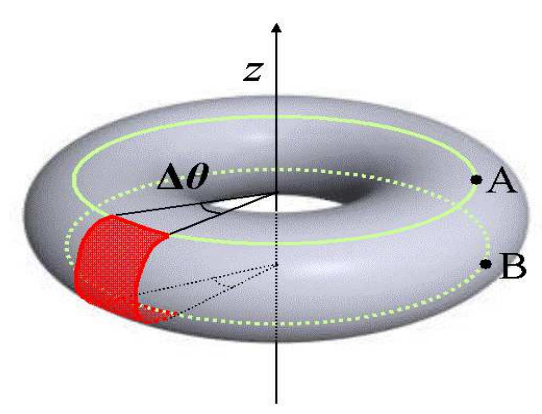

FIG. 2: Patch of positive Gaussian curvature on the torus (shaded) subtending an azimuthal angle $\Delta \theta$. The surface of the torus is divided into regions of positive and negative Gaussian curvature by the circles labelled $\mathrm{A}$ and $\mathrm{B}$, on which the Gaussian curvature vanishes (after D. Hilbert and S. CohnVossen, Geometry and the Imagination (Chelsea, New York, 1952).

The "electrostatic" energy term in Eq.(4) favors approximately charge neutral configurations, with discrete disclination charges cancelling the smeared out Gaussian "curvature charge". Although the full calculation (with core energies taken into account) is subtle (see Sec IV], it is interesting to estimate how many additional disclinations might be accommodated on a torus. In the torus shown in Fig 2 the solid and dashed circles divide the surface into regions of positive and negative Gaussian curvature. Consider a wedge of angular width $\Delta \theta$ on the outside wall of positive Gaussian curvature. The net "curvature charge" associated with this region is

$$
\Delta K=\int_{0}^{\Delta \theta} d \theta \int_{-\pi / 2}^{\pi / 2} d \alpha \sqrt{g} K(\alpha)=2(\Delta \theta)
$$

where we have used Eq.(7) and $\sqrt{g}=$ $R_{1} R_{2}\left(1+\frac{R_{2}}{R_{1}} \cos \alpha\right)$. Upon equating $\Delta K$ to $2 \pi / 6$, the charge of a single disclination, we find that $\Delta \theta=2 \pi / 12$, independently of $R_{1}$ and $R_{2}$. Thus $2 \pi / \Delta \theta=12$ positive disclinations would be required to completely compensate the curvature of the outer wall. Similarly, 12 negative disclinations would be required to completely compensate the negative curvature of the inner wall. This simple argument neglects core energies and interactions between disclinations, effects which will 
cause the preferred number of defect pairs to be less than 12.

The thermodynamic limit for torus dimensions $R_{1}$ and $R_{2}$ corresponds to the limits $R_{1} / a_{0} \rightarrow \infty$ and $R_{2} / a_{0} \rightarrow$ $\infty$, with the aspect ratio $r=R_{1} / R_{2}$ fixed, where $a_{0}$ is a microscopic length scale such as the particle spacing. Upon optimizing $r$ with a defect-free hexatic texture on the torus, one finds that a nonzero hexatic stiffness constant $K_{A}$ pulls $r$ above the Clifford torus value $r=\sqrt{2}$ appropriate for liquid torii [11], so that the resulting shape looks more like a bicycle tire (see Sec. IV.A). Our results for defect energies are presented for fixed $r$ and a given number of $M \sim R_{1} R_{2} / a_{0}^{2}$ of microscopic degrees of freedom. It would be straightforward, however, to use the methods described here to optimize over both $r$ and possible defect configurations for fixed $M$.

Although we find that disclinations are always unfavorable in the "thermodynamic limit" of large $M$, the critical value $M=M_{c}$ required to suppress them in the ground state is surprisingly large. Indeed, for $r=\sqrt{2}$, this number is of order $10^{11}$ ! (see Fig 12). As $r$ approaches 1 from above, i.e., in the limit of "fat" torii, we find that $M_{c}$ exhibits a remarkably strong divergence [see Eq.(49)],

$$
M_{c} \sim \frac{1}{(r-1)^{12}},
$$

as treated in Sec. IV.C. Because $M_{c}$ is so large, the toroidal vesicles of Ref. [1] would be quite likely to have disclinations present in the ground state if hexatic order were present. Indeed, for $R_{1}=5 \mu$ (roughly the size of a red blood cell), $R_{2}=r R_{1} \approx 1.4 R_{1}$ and $a_{0}=20 \AA$ (typical lipid spacing in vesicles), we have (see Sec.IV]-B) $M=\frac{8 \pi^{2}}{\sqrt{3}} \frac{R_{1} R_{2}}{a_{0}^{2}} \approx 4 \times 10^{8}$ which is much less than the critical value $M_{c} \sim 10^{11}$ required to suppress disclinations. As discussed above, the interaction between hexatic order and the Gaussian curvature of toroidal vesicles leads to $r>\sqrt{2}$ and a smaller value of $M_{c}$. Vesicles with lipids in a liquid state could provide, however, a toroidal template with $r=\sqrt{2}$ for adsorbed colloidal particles, similar to the spherical "colloidosomes" studied by Dinsmore et. al. [12. It may be possible to use polymerizable lipids to permanently fix the template aspect ratio at $r=\sqrt{2}$. It would be quite interesting to study (with, say, confocal microscopy) both hexatic and crystalline order in colloidal particle arrays [13] adsorbed on such a template, as has already been done for colloids on spherical water droplets in oil [14]. The colloid radius would play the role of a microscopic scale $a_{0} \ll R_{1}, R_{2}$ in this case. Disclination defects in a crystalline ground state might well be accompanied by grain boundaries [19.

Although we focus here on hexatic order in toroidal geometries, similar results should apply to XY-like models, as would be appropriate for vesicles composed of lipid bilayers with tilted molecules [3, 4]. Our results are relevant as well to two-fold nematic order on a toroidal template. In both cases, we expect qualitatively similar phenomenon, such as defects in the ground state, unless the total number of degrees of freedom exceeds a critical value. A precise equivalence is possible in the one Frank constant approximation 15. As discussed in Sec. IV defects in the ground state are more likely for fat torii in the case of nematic (and hexatic) order. Interesting results related to those here have recently been obtained for "corrugated" topographies, which are flat at infinity and for which there is also no topological necessity for defects in the ground state [16]. Specifically, it has been shown that defect pairs lower the energy of a hexatic phase draped over a Gaussian "bump" for sufficiently large ratio of height to width. In this case, defects remain an important feature of the ground state even when the number of degrees of freedom $M$ tends to infinity.

The organization of the paper is as follows: an analogy to an electrostatic problem, aimed at providing a more intuitive picture of the physics of curvature-induced defect unbinding, is introduced in Sec. [II In Sec. III the interaction among defects is worked out in detail for toroidal topology. Predictions for the total number of defects are provided in Sec. IV The effects of both temperature and shape fluctuations are discussed in Sec. $\nabla$

\section{ELECTROSTATIC ANALOGY}

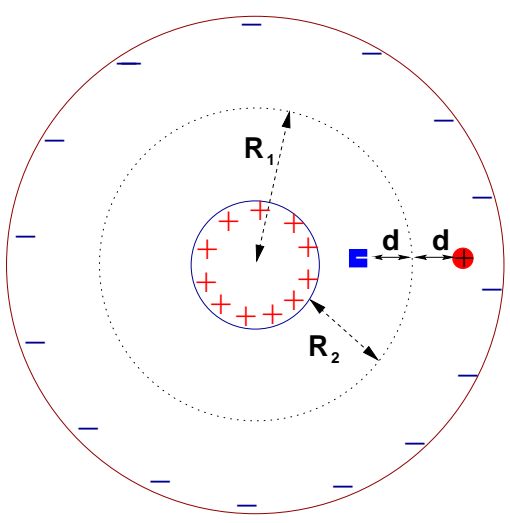

FIG. 3: A plus (filled circle) and a minus (filled square) pair of charges between the plates of a two-dimensional circular capacitor is analogous to a plus-minus pair of disclinations on a torus with the identifications given.

It is useful to illustrate the physics of defects on the torus with a simple electrostatic analogy, illustrated in Fig. 3. A positive and negative charge are placed between the plates of a capacitor with circular cross section. The plus-minus pair are the analogs of a plus-minus disclination dipole initially located on a zero curvature line of the torus (see Fig. (4). We assume here that the charges are extended over a core radius $a_{0}$, which plays the role of a minimum separation. We expect that this core radius is related to the mean particle separation on the torus. The net charge $+Q$ and $-Q$ on the capacitor plates represents the Gaussian curvature in Eq.(4), integrated over the regions of the torus where it is positive 
and negative, respectively. The linear charge density on the plates of the capacitor is thus given by $\rho_{ \pm}=Q / L_{ \pm}$, where $L_{ \pm}=2 \pi\left(R_{1} \mp R_{2}\right)$. The lengths $R_{1}$ and $R_{2}$ for this capacitor correspond to the torus radii defined in Fig. 1

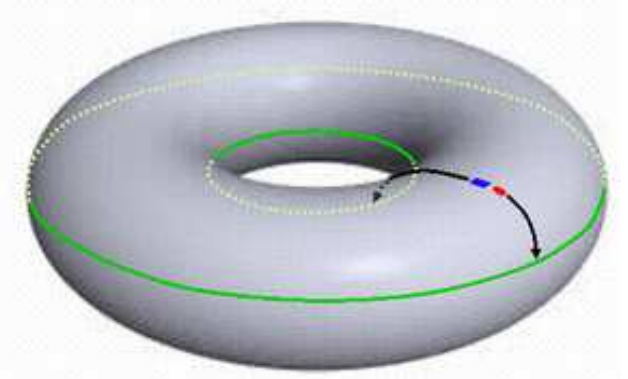

FIG. 4: Illustration of the calculation discussed in the text: a plus (filled circle) and a minus (filled square) form a disclination dipole on one of the two circles of zero Gaussian curvature. They are then pulled apart until they reach the maximum curvature line (plus) and minimum curvature line (minus).

The competition between the work done by the electric field of the capacitor (representing regions of +/- Gaussian curvature on the torus) and the attraction of opposite sign charges will dictate whether the disclinations separate or remain tightly bound at separation $a$. Since the energy of a configuration with excess bound charges exceeds that for no excess charges by an amount of order two core energies, this criterion determines whether a separated plus-minus pair is present in the ground state.

In this two-dimensional geometry, the electrostatic energy of the two charges with separation $2 d$ and charge $e$ is given by

$$
\mathcal{E}_{1}=e^{2} \ln \left(d / a_{0}\right)+2 E_{c}
$$

where $E_{c}$ represents a self-energy of an isolated charge, corresponding to the core energy of a disclination. There is an additional electrostatic force pulling the charges to the capacitor plates, which leads to an additional energy

$$
\mathcal{E}_{2}=Q e \ln \left(\frac{R_{1}-d}{R_{1}+d}\right)
$$

The total energy is then

$$
\mathcal{E}=2 \pi e^{2} \mathcal{Q}-Q e \mathcal{L}+2 E_{c},
$$

where

$$
\begin{aligned}
\mathcal{Q} & =\frac{1}{2 \pi} \ln \left(d / a_{0}\right), \\
\mathcal{L} & =\ln \left(\frac{R_{1}+d}{R_{1}-d}\right) .
\end{aligned}
$$

The functions $\mathcal{Q}$ and $\mathcal{L}$ have a similar form to those we find in the exact calculation on the torus (see Eq.(31)).
Eq. (13) illustrates very clearly the appearance of two preferred locations; these being $d=a_{0}$ (where $\left.\mathcal{E} \approx 2 E_{c}\right)$ and $d=R_{2}$ $\left(\right.$ where $\left.\mathcal{E}=2 E_{c}+e^{2} \ln \left(R_{2} / a_{0}\right)-e Q \ln \left[\frac{R_{1}+R_{2}}{R_{1}-R_{2}}\right]\right)$. The relative strength of the first two terms will determine the preferred location of the charge dipole. By taking $a_{0}$ small enough, for $R_{1}>R_{2}$, the attractive charge-charge term will dominate and defects will not be favored. For any finite $a_{0}$, on the other hand, defect unbinding will be favored in the limit $R_{2} \rightarrow R_{1}^{-}$. This is precisely what we find, up to numerical constants, in our treatment of disclination unbinding on the torus.

\section{INTERACTING DEFECTS ON CURVED SURFACES}

\section{A. Green's function on the Torus}

For arbitrary coordinates $\overrightarrow{\mathbf{x}}=\left(x_{1}, x_{2}\right)$ on the torus, the key object in the energy Eq. (4) is the inverse Laplacian, which is the solution $G\left(\overrightarrow{\mathbf{x}}, \overrightarrow{\mathbf{x}^{\prime}}\right)$ to the equation

$$
\Delta G\left(\mathbf{x}, \mathbf{x}^{\prime}\right)=\delta\left(\mathbf{x}, \mathbf{x}^{\prime}\right)-\frac{1}{A},
$$

where the Laplacian is defined as in Eq.(5),$\delta\left(\mathbf{x}, \mathbf{x}^{\prime}\right)=$ $\frac{1}{\sqrt{g}} \delta\left(\mathbf{x}-\mathbf{x}^{\prime}\right)$, and $A$ is the area of the torus,

$$
A \equiv \int d^{2} \mathbf{x} \sqrt{g}=4 \pi^{2} R_{1} R_{2} .
$$

A constant is subtracted from the $\delta$-function to eliminate a "zero mode" which changes the area of the torus. As shown in the Appendix, the metric of a torus, with a suitable change of coordinates, is flat modulo an overall conformal factor [see Eq.(65)]. The inverse Laplacian may be computed by considering the flat metric (where the conformal factor is identically one) and its associated inverse Laplacian $\widehat{G}\left(\mathbf{x}, \mathbf{x}^{\prime}\right)$, from the formula

$$
\begin{aligned}
G\left(\mathbf{x}, \mathbf{x}^{\prime}\right) & =\widehat{G}\left(\mathbf{x}, \mathbf{x}^{\prime}\right) \\
& -\quad \int \frac{d^{2} \mathbf{y}}{A} \sqrt{g(\mathbf{y})}\left[\widehat{G}(\mathbf{x}, \mathbf{y})+\widehat{G}\left(\mathbf{y}, \mathbf{x}^{\prime}\right)\right] \\
& +\quad \int \frac{d^{2} \mathbf{y}}{A} \int \frac{d^{2} \mathbf{y}^{\prime}}{A} \sqrt{g(\mathbf{y})} \sqrt{g\left(\mathbf{y}^{\prime}\right)} \widehat{G}\left(\mathbf{y}, \mathbf{y}^{\prime}\right)
\end{aligned}
$$

As can be checked straightforwardly, $G\left(\mathbf{x}, \mathbf{x}^{\prime}\right)$ solves Eq. (15) as well as satisfying

$$
\begin{gathered}
\int d^{2} \mathbf{x} \sqrt{g(\mathbf{x})} G\left(\mathbf{x}, \mathbf{x}^{\prime}\right)=0 \\
\int d^{2} \mathbf{x}^{\prime} \sqrt{g\left(\mathbf{x}^{\prime}\right)} G\left(\mathbf{x}, \mathbf{x}^{\prime}\right)=0 .
\end{gathered}
$$

Thus Eq.(17) is indeed the inverse Laplacian, where the conditions (18) ensure overall "charge neutrality" for any disclinations present on the torus. 
The coordinates for the torus are shown in Fig. 1 Upon making the change of variables $\alpha \rightarrow \varphi$ via $\cos \alpha \equiv \frac{R_{1} \cos \varphi-R_{2}}{R_{1}-R_{2} \cos \varphi}$, described in the Appendix, the inverse Laplacian $\widehat{G}\left(\mathbf{x}, \mathbf{x}^{\prime}\right)$ in conformal coordinates can be computed by first solving

$$
\begin{array}{r}
-\left(\sinh \rho \partial_{\theta}^{2}+\frac{1}{\sinh \rho} \partial_{\varphi}^{2}\right) \widehat{G}\left(\theta, \varphi \mid \theta^{\prime}, \varphi^{\prime}\right)= \\
\delta\left(\theta-\theta^{\prime}, \varphi-\varphi^{\prime}\right)-\frac{1}{(2 \pi)^{2}}
\end{array}
$$

where $\sinh \rho=\sqrt{r^{2}-1}$ and $r$ is the aspect ratio $R_{1} / R_{2}$ of the torus. The straight-forward solution

$$
\begin{array}{r}
\widehat{G}^{(0)}\left(\theta, \varphi \mid \theta^{\prime}, \varphi^{\prime}\right)= \\
-\frac{1}{4 \pi} \ln \left[(\sinh \rho)\left(\theta-\theta^{\prime}\right)^{2}+\frac{1}{\sinh \rho}\left(\varphi-\varphi^{\prime}\right)^{2}\right],
\end{array}
$$

satisfies $\Delta \widehat{G}^{(0)}=\delta\left(\theta-\theta^{\prime}, \varphi-\varphi^{\prime}\right)$, but is not periodic, i.e.,

$$
\begin{aligned}
\widehat{G}^{(0)}\left(\theta+2 \pi k, \varphi \mid \theta^{\prime}, \varphi^{\prime}\right) & \neq \widehat{G}^{(0)}\left(\theta, \varphi+2 \pi n \mid \theta^{\prime}, \varphi^{\prime}\right) \\
& \neq \widehat{G}^{(0)}\left(\theta, \varphi \mid \theta^{\prime}, \varphi^{\prime}\right),
\end{aligned}
$$

for arbitrary integers $k$ and $n$. In addition the Laplacian acting on $\widehat{G}^{(0)}\left(\theta, \varphi \mid \theta^{\prime}, \varphi^{\prime}\right)$ fails to give the constant term on the right hand side of Eq.(19). Both these deficiencies are remedied by defining

$$
\begin{aligned}
\widehat{G}\left(\theta, \varphi \mid \theta^{\prime}, \varphi^{\prime}\right) & =-\frac{1}{2 \pi} \sum_{k, n} \ln \left[\sinh \rho(\theta+2 \pi k)^{2}\right. \\
& \left.+\frac{1}{\sinh \rho}(\varphi+2 \pi n)^{2}\right]+\mathcal{C}(\rho),
\end{aligned}
$$

where the constant $\mathcal{C}(\rho)$ is determined by imposing that the inverse Laplacian exhibits the correct short-distance singularity (following from Eq.(201) ) in the limit $\theta \rightarrow \theta^{\prime}$ and $\varphi \rightarrow \varphi^{\prime}$. Standard analytical techniques [22, 23] allow one to perform the sum indicated in Eq. (22), giving

$$
\begin{aligned}
\widehat{G}\left(\theta, \varphi \mid \theta^{\prime}, \varphi^{\prime}\right) & =-\frac{1}{4 \pi} \ln \left[\frac{\sinh \rho\left|\vartheta_{1}\left(\frac{\theta-\theta^{\prime}+\tau\left(\varphi-\varphi^{\prime}\right)}{2 \pi}, \tau\right)\right|^{2}}{4 \pi^{2}|\eta(\tau)|^{6}}\right] \\
& +\frac{1}{2 \sinh \rho}\left(\frac{\varphi-\varphi^{\prime}}{2 \pi}\right)^{2},
\end{aligned}
$$

where $\tau=\frac{i}{\sinh \rho}$. The functions $\vartheta_{1}$ and $\eta$ are the Theta function and Dedekind $\eta$ function, respectively, defined by 24$]$

$$
\vartheta_{1}(\nu, \tau)=-i \sum_{n=-\infty}^{n=+\infty}(-1)^{n} e^{i \pi \tau(n-1 / 2)^{2}} e^{2 \pi i \nu(n-1 / 2)}
$$

and

$$
\eta(\tau)=e^{2 \pi i \tau / 24} \prod_{n=1}^{\infty}\left(1-e^{2 \pi i n \tau}\right) .
$$

The inverse Laplacian of Eq. (17) thus becomes

$$
\begin{gathered}
G\left(\theta, \varphi \mid \theta^{\prime}, \varphi^{\prime}\right)=\widehat{G}\left(\theta, \varphi \mid \theta^{\prime}, \varphi^{\prime}\right)- \\
\frac{2}{(2 \pi)^{2}}\left(\frac{1}{\sinh \rho} \sum_{n=1}^{\infty} \frac{e^{-n \rho}}{n^{2}}\left[\cos (n \varphi)+\cos \left(n \varphi^{\prime}\right)\right]\right. \\
\left.+\frac{1}{\cosh \rho} \sum_{n=1}^{\infty} \frac{e^{-n \rho}}{n}\left[\cos (n \varphi)+\cos \left(n \varphi^{\prime}\right)\right]\right)+ \\
+\frac{2}{(2 \pi)^{2}}\left(\frac{1}{\sinh \rho} \sum_{n=1}^{\infty} \frac{e^{-2 n \rho}}{n^{2}}+\right. \\
\left.\frac{2}{\cosh \rho} \sum_{n=1}^{\infty} \frac{e^{-2 n \rho}}{n} \frac{\tanh \rho}{\cosh \rho} \sum_{m=1}^{\infty} e^{-2 m \rho}\right)
\end{gathered}
$$

which can then be used to evaluate Eq. (4).

\section{B. Energetics of Defects on a Torus}

The total energy Eq.(4) also contains a bending rigidity term which, for a torus with aspect ratio $r=R_{1} / R_{2}$, is

$$
\mathcal{E}^{\kappa}=\frac{2 \pi^{2} r^{2}}{\sqrt{r^{2}-1}} \kappa
$$

If we were to minimize this term alone, we would find $r=\sqrt{2}$, the so-called Clifford torus. The Gauss-Bonnet theorem for a torus reads

$$
\int d^{2} \mathbf{x} \sqrt{g} K(\mathbf{x})=0
$$

and, with our choice of Green's functions, Eq.(1) insures that the sum of disclination "charges" $q_{i}$ satisfies

$$
\sum_{i=1}^{N} q_{i}=0
$$

so that, as previously noted, no defects are required topologically. The defect charges here take on the values $q_{i}= \pm 1, \pm 2, \ldots$, with $q_{i}= \pm 1$ corresponding to the elementary defects with rotations $\pm 2 \pi / 6$ in the hexatic order parameter.

With the Green's function in hand, the hexatic energy of a set of disclination charges on a torus (the first part of Eq.(10) can thus be written explicitly:

$$
\begin{aligned}
\mathcal{E} & =\frac{\pi^{2} K_{A}}{18} \sum_{i \neq j}^{N} q_{i} q_{j} \mathcal{Q}\left(\mathbf{x}_{i}, \mathbf{x}_{j}\right)-\frac{\pi K_{A}}{3} \sum_{i=1}^{N} q_{i} \mathcal{L}\left(\mathbf{x}_{i}\right) \\
& +\mathcal{D}+\left(\sum_{i=1}^{N} q_{i}^{2}\right) E_{c}
\end{aligned}
$$

where the defects interact with each other according to

$$
\begin{aligned}
\mathcal{Q}\left(\mathbf{x}_{i}, \mathbf{x}_{j}\right) & =-\frac{1}{4 \pi} \ln \left(\frac{\left|\vartheta_{1}\left(\frac{\theta_{i}-\theta_{j}}{2 \pi}+\frac{i\left(\varphi_{i}-\varphi_{j}\right)}{2 \pi \sinh \rho}, \frac{i}{\sinh \rho}\right)\right|^{2}}{4 \pi^{2}\left|\eta\left(\frac{i}{\sinh \rho}\right)\right|^{6}}\right) \\
& +\frac{1}{2 \sinh \rho}\left(\frac{\varphi_{i}-\varphi_{j}}{2 \pi}\right)^{2}
\end{aligned}
$$


and interact with the background Gaussian curvature "charge" according to

$$
\mathcal{L}(\mathbf{x})=\ln \left(\frac{1}{\cosh \rho-\cos \varphi}\right) .
$$

The "spin wave" part of the frustrated hexatic energy

$$
\mathcal{D}=\frac{1}{2} K_{A}(2 \pi)^{2} e^{-\rho}=\frac{2 \pi^{2} K_{A}}{r+\sqrt{r^{2}-1}}
$$

is present even without defects and supplements the bending rigidity term Eq. (27). The core energy term in Eq. (30) will be considered in more detail in Sec. IV

\section{Energetics of Defects on a Sphere}

It is instructive to compare our results for toroidal vesicles with the corresponding results for spherical vesicles. The interaction potential (31) for a sphere is

$$
\mathcal{Q}\left(\mathbf{x}_{i}, \mathbf{x}_{j}\right)=-\frac{1}{4 \pi} \ln \left(\frac{1-\cos \beta_{i j}}{2}\right)
$$

where $\beta_{i j}$ is the geodesic distance, for a sphere of unit radius, between points $\mathbf{x}_{i}$ and $\mathbf{x}_{j}$. The function $\mathcal{L}(\mathbf{x})$ and the constant $\mathcal{D}$ can both be set to zero on the sphere.

The Gauss-Bonnet theorem for spherical topology reads

$$
\int d^{2} \mathbf{x} \sqrt{g} K(\mathbf{x})=4 \pi,
$$

yielding the constraint

$$
\sum_{i=1}^{N} q_{i}=12 .
$$

\section{GROUND STATES OF HEXATIC TOROIDAL VESICLES}

Before presenting a detailed analysis of the implications of the Hamiltonian (30) for defects on a torus we must confront the issue of core energies. The shortdistance behavior of the defect potential $\mathcal{Q}$ (Eq.(31) ) implies that a plus-minus pair of disclinations located as in Fig 4 on a circle of fixed azimuthal angle $\theta$ and geodesic separation $2 d$ have an energy

$$
E=\frac{K_{A} \pi}{18}\left[\ln \left(R_{2} / a_{0}\right)+V_{I}\left(d / R_{2}\right)\right]+2 E_{c},
$$

where, after absorbing various constants, $V_{I}\left(d / R_{2}\right)$ may be viewed as the interaction potential between the two disclinations and the term $E_{c}$ (reflecting short-distance physics) has been added by hand. For $a_{0} \ll 2 d \ll R_{2}$, $V_{I}\left(d / R_{2}\right) \simeq \ln \left(2 d / R_{2}\right)$. The torus radius $R_{2}$ therefore drops out in this limit and we recover the result $E=$
$K_{A} \pi / 18 \ln \left(2 d / a_{0}\right)+2 E_{c}$ for a disclination pair in flat space. The disclination core radius $a_{0}$ is the distance (of order the spacing between molecules) at which continuum elasticity breaks down. We will henceforth assume that

$$
E_{c} \approx c K_{A},
$$

where $c$ is a dimensionless parameter characterizing the size of the disclination core energy. In our numerical calculations we take $c=0.1$.

If the torus is coated with $M$ particles, an effective average particle spacing $a_{P}$ (assuming a local triangular lattice for simplicity) may be defined from the area per particle

$$
\frac{\sqrt{3}}{2} a_{P}^{2}=\frac{4 \pi^{2} R_{1} R_{2}}{M}=\frac{4 \pi^{2} R_{2}^{2} r}{M} .
$$

We shall assume that $a_{0} \approx a_{P}$, so Eq. (39) relates the total number of particles $M$ to the minimum plus-minus pair separation appearing in Eq. (37).

Upon including both the bending rigidity and the core energies, the total energy for $N$ charge \pm 1 defects on a rigid, undeformed torus takes the form

$$
\begin{aligned}
& \mathcal{E}=N E_{c}+\frac{K_{A}}{2}\left(\frac{2 \pi^{2}}{9} \sum_{i=1}^{N} \sum_{j>i}^{N} q_{i} q_{j} \mathcal{Q}\left(\mathbf{x}_{i}, \mathbf{x}_{j}\right)\right. \\
& \left.-\frac{2 \pi}{3} \sum_{i=1}^{N} q_{i} \mathcal{L}\left(\mathbf{x}_{i}\right)+\frac{4 \pi^{2}}{r+\sqrt{r^{2}-1}}\right)+\kappa \frac{2 \pi^{2} r^{2}}{\sqrt{r^{2}-1}}(40)
\end{aligned}
$$

$\mathcal{E}$ depends on the lattice spacing $a_{0}$ through the core energy Eq. (38) as well as the constraint that the defect spacing cannot be smaller than $a_{P}$. In our calculations the latter constraint is accounted for by forbidding any two defects from approaching within the distance $a_{P}$ or, alternatively, by assuming that any two defects closer than $a_{P}$ merge into a single defect with total charge the sum of the individual charges of the two defects.

Only the potential $(\mathcal{Q})$ and curvature $(\mathcal{L})$ terms depend explicitly on the defect positions and charges. For fixed $R_{1}$ and $R_{2}$, therefore, the total number of defects in the ground state is independent of both the bending rigidity and the constant $\mathcal{D}$ in Eq. (333).

The defect-defect interaction is determined through the $\mathcal{Q}$-function defined in Eq. (31). For two opposite sign defects the energy is attractive for all separations at constant $\theta$, as shown in Fig. [5 If only this term were present, the attraction would bring both charges as close as possible, binding all disclinations into dipoles which have a higher energy than a defect-free configuration. Thus if no other terms were present, the ground state would be defect free.

The defect-curvature interaction $\mathcal{L}$ favors the appearance of additional defects. This term acts like an electric field pulling the positive/negative disclinations into regions of positive/negative Gaussian curvature, respectively, similar to the electrostatic analogy discussed in 


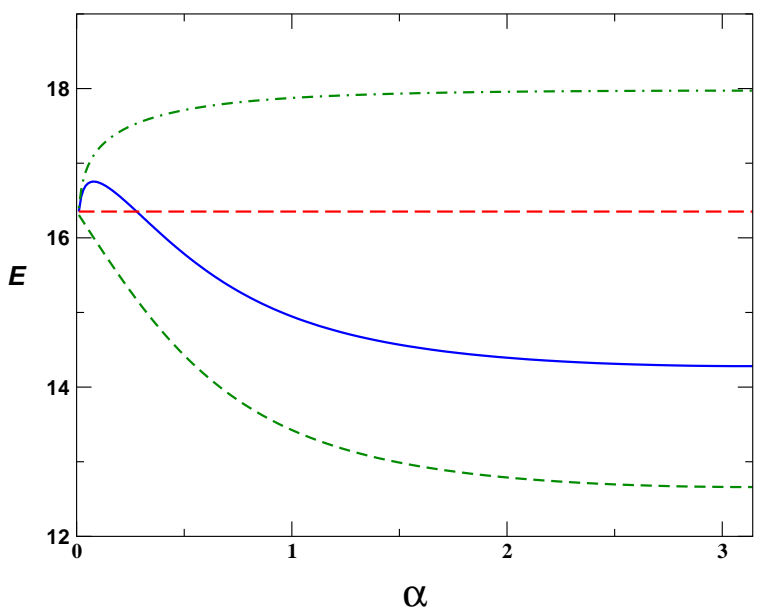

FIG. 5: Various contributions to the energy (in units of $K_{A} / 2$ ) with aspect ratio $r=\sqrt{2}$ for a disclination dipole separated an angular distance $\alpha$ along the path shown in Fig. 4 The long dashed line is the energy in the absence of defects. The dashed-dotted line is the defect-defect interaction as a function of the separation of the charges. The dashed line is the curvature-defect interaction energy as a function of separation, and the continuous line is the total energy. The core energy contribution, computed from Eq. 38 is very small on this scale.The bending energy is subtracted for clarity.

Sec II As shown in Fig. [5 if this were the only term present, isolated plus and minus disclination charges are always energetically favored, with the lowest energy arising when they are located at the regions of absolute maximum Gaussian curvature.

The total energy is a competition between defect attraction and curvature-induced unbinding, as shown in Fig. 5 where the potential shows a double well, corresponding to the attractive defect-defect interaction minimum and the attractive Gaussian curvature minimum. In Fig. 5 the curvature field dominates and additional defects appear in the ground state, but the general result for whether defects should or should not be expected is a function of the core energy $E_{c}$, the hexatic stiffness $K_{A}$, the torus aspect ratio $r=R_{1} / R_{2}$, and the ratio $R_{2} / a_{P}$ of macroscopic to microscopic cutoff.

\section{A. Defect-free hexatic toroidal vesicles}

In the absence of defects the total energy following from Eq. (30) is

$$
E=\frac{2 \pi^{2} K_{A}}{r+\sqrt{r^{2}-1}}+\kappa \frac{2 \pi^{2} r^{2}}{\sqrt{r^{2}-1}}
$$

a result first obtained in 11]. The optimal value of $r$ resulting from minimizing this energy is the solution of

$$
\left(r^{2}-1\right)^{3 / 2}-r\left(r^{2}-1\right)+\frac{\kappa}{K_{A}} r\left(r^{2}-2\right)=0
$$

which read in the limit of large and small hexatic stiffness [1],

$$
\begin{aligned}
& r=\sqrt{2}, K_{A}<<\kappa \\
& r=\sqrt{\frac{K_{A}}{2 \kappa}}, K_{A}>>\kappa .
\end{aligned}
$$

If the hexatic stiffness is much smaller than the bending rigidity, the Clifford torus $(r=\sqrt{2})$ is the optimal geometry. If, on the other hand, the hexatic stiffness dominates, then a thin torus, similar to a bicycle tire, is optimal. This picture changes when defects are included.

\section{B. Ground states of defective hexatic toroidal vesicles}

The general ground state for arbitrary aspect ratio may be determined numerically using Eq. (40). For simplicity, we compare the energies of configurations with and without defects for a fixed aspect ratio $r$.

We first performed the following calculation: a set of $N$ unit charge disclinations $(N / 2$ positive and $N / 2$ negative) are placed in opposite-sign pairs on a circle of zero Gaussian curvature. Each pair is then pulled apart at constant azimuthal angle $\theta$ until the plus(minus) disclinations reach the outer(inner) rim of the torus respectively. This is illustrated in Fig. 4 for the simplest case $N=2$.

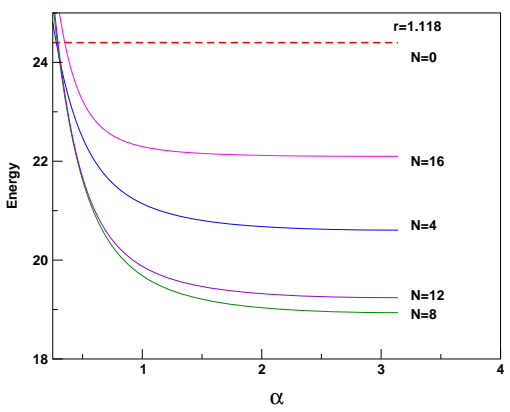

FIG. 6: The total energy (in units of $K_{A} / 2$ ) for aspect ratio $r=\sqrt{5 / 4}$ for varying numbers of defects and $a_{P}=R_{2} / 4$. The bending energy at fixed $r$ is subtracted off. The disclination core energy is taken to be $0.1 K_{A}$, which is 0.2 in the above units (corresponding to $c=0.1$ ).

We discuss our results for two regimes of disclination core energy. Core energies corresponding to $c$-coefficients less than $1 / 10$ have almost no effect on the energy balance since the elastic energy is already quite large. For definiteness the small core energy regime will be illustrated for $c=\frac{1}{10}$. Our results are shown in Figs. 6 [7] and 8 for the three aspect ratios: $r=\sqrt{5 / 4}, r=\sqrt{2}$ (the Clifford torus) and $r=2.69$. We have set $R_{2} / a_{P}=4$, so that the number of degrees of freedom (see Eq. (39)) on these torii is $M=\frac{8 \pi^{2}}{\sqrt{3}} r\left(\frac{R_{2}}{a_{P}}\right)^{2} \simeq 800,1000$ and 2000 


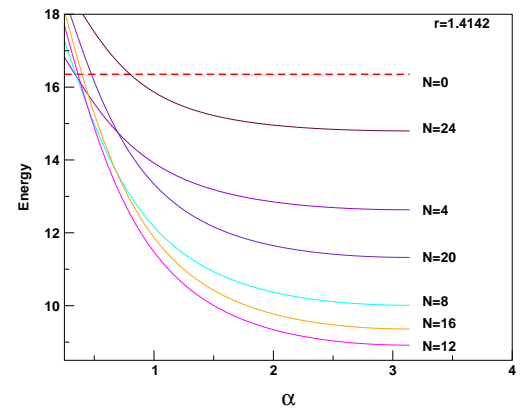

FIG. 7: The total energy, as in Fig. 6] but for the Clifford torus which has aspect ratio $r=\sqrt{2}$.

respectively. In each case the addition of defects lowers the energy. Note that the energy at maximum separation $(\alpha=\pi)$ first decreases, and then increases with $N$. The optimal number of defect pairs $(N / 2)$ is 5,6 and 7 for Figs. 6 7 and 8 respectively. This number is less than the naive estimate of 12 in the Introduction due to repulsive defect interaction energies on the inner and outer walls of the torus.

The typical situation for large core energies is illustrated for $c=1$. It is only for core energies of this order that we find qualitatively different behavior from the small core energy regime. Our results are plotted in Fig. 9 $(r=\sqrt{5 / 4})$ and Fig. 10 $(r=\sqrt{2})$. Because $R_{2} / a_{P}=\frac{100}{\pi} \simeq 32$ in these plots, we now have $M \approx 52,000$ and $M \approx 67,000$ respectively. Even with such a large core energy, defects are present in the ground state with a preferred number of pairs $N^{*} / 2 \simeq 3$ for Fig. 9 and a smaller number for Fig. 10, With this value of $R_{2} / a_{P}$ the torus is defect free for larger $r$.

Note that for fixed aspect ratio the preferred number of defects drops for larger numbers of particles when the core energy is large: compare Fig. 9 to Fig. 7 To study this point further we have determined the total number of defects in the ground state as a function of the number of particles. Our results are shown in Fig. [1] using $M=\frac{8 \pi^{2}}{\sqrt{3}} r\left(R_{2} / a_{P}\right)^{2}$ and assuming $a_{0}=a_{P}$. Although a torus always becomes defect free in the thermodynamic limit $R_{2} / a_{P} \rightarrow \infty$ (with $r=R_{1} / R_{2}$ fixed),

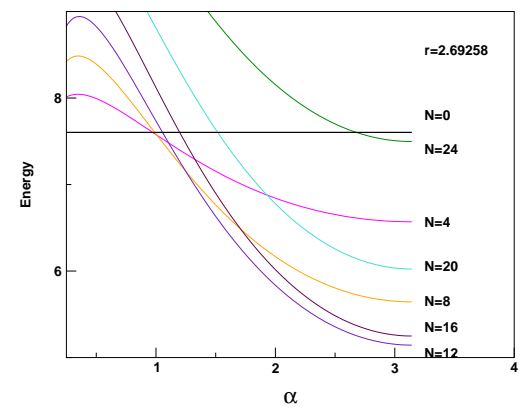

FIG. 8: The total energy, as in Fig. 6] but for aspect ratio $r=2.6926$.

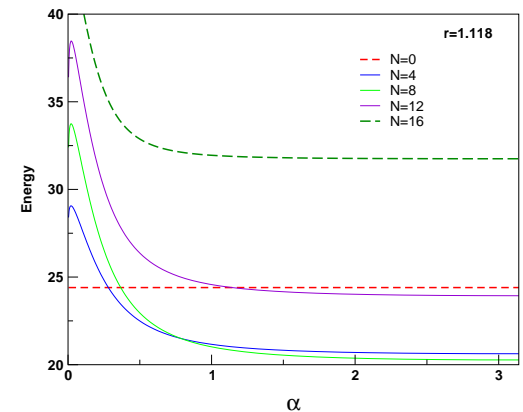

FIG. 9: The total energy (in units of $K_{A} / 2$ ) for a torus with aspect ratio $r=\sqrt{5 / 4}$ for varying numbers of defects with $R_{2} / a_{P}=100 / \pi$. The disclination core energy is taken to be $K_{A}$ which is 2 in the above units (corresponding to $c=1$ ). The bending energy at fixed $r$ is subtracted off.

torii with moderate aspect ratio only become defect-free for a remarkably large number of particles, which may be as large as $10^{11}$ for $r=\sqrt{2}$ !

To make the last point more transparent, the critical number of particles $M_{c}$, above which defects are no longer favored, is plotted as a function of the aspect ratio in Fig. 12. As the aspect ratio $r=R_{1} / R_{2} \rightarrow 1^{+}, M_{c}$ diverges, suggesting that any torus will possess defects if sufficiently fat. We provide an rough analytic argument along this line in the next subsection.

Although fat torii (with $r \gtrsim 1$ ) tend to favor defects in the ground state, the maximum number of defects favored for a given aspect ratio is a subtle question. To see this, note that the constrained minimization discussed above leads to a ring of $N / 2$ positive disclination charges on the outer wall of the torus, and a smaller ring of $N / 2$ negative charges on the inner wall. As $r \rightarrow 1^{+}$, negative charges end up being very close in the final configuration, with a considerable repulsive energy cost.

To allow the system to reduce this energy, we have considered the modified calculation illustrated in Fig. 13. The initial configuration starts from both circles of zero curvature, alternating plus-minus defect pairs between them. When the separation variable $\alpha=\pi$ the configuration is the same as in the previous case. A typical

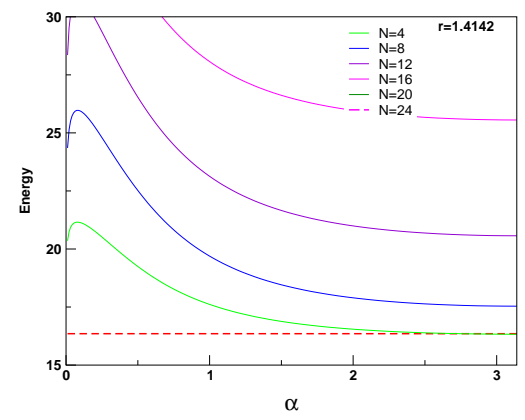

FIG. 10: The total energy, as in Fig. 9 but for aspect ratio $r=\sqrt{2}$. 


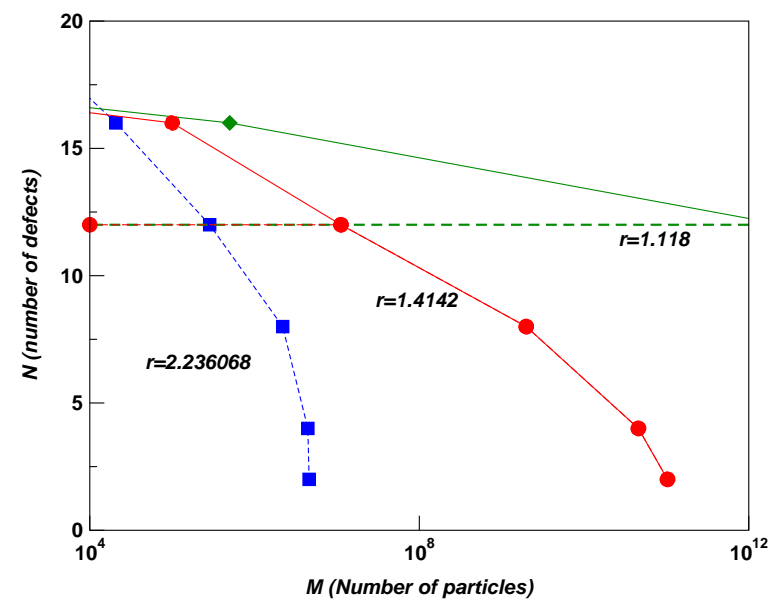

FIG. 11: The preferred number of defects as a function of the total number of particles for three aspect ratios. The disclination core energy is taken to be $0.1 K_{A}$. The dashed line corresponds to the configuration in Fig. 4

plot of the energy is shown in Fig. 14 In this plot, the minimal configurations (with $\alpha \neq \pi$ ) correspond to buckled or staggered rings of defects, displaced from the circles of maximum or minimum curvature, as illustrated in Fig. 15. The final position is a compromise between the "electrostatic" repulsion and the attraction to the Gaussian curvature basins. With the extra degrees of freedom allowed by buckling, the energies are always lower than the simple ring configurations of Fig. [6] and Fig. 9 Note that staggering allows more defects to be squeezed into the ground state: the optimal number $N^{*} / 2$ of defect pairs is 7 in Fig. [14 as opposed to $N / 2 \approx 4-5$ in Fig. [6]

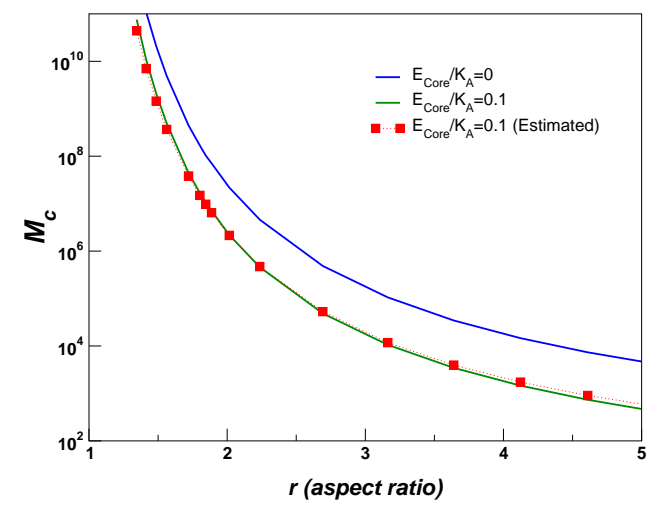

FIG. 12: The critical number of particles, above which defects are no longer favored, as a function of the toroidal aspect ratio for vanishing core energy (blue) and $c=0.1$ (green). The analytic estimate for $c=0.1$ is plotted as solid red squares.

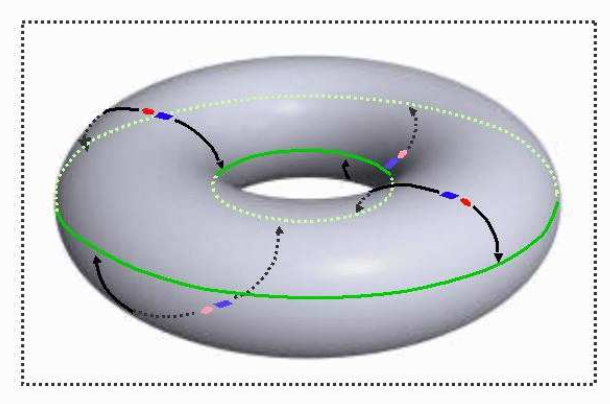

FIG. 13: Illustration of the calculation discussed in the text: a plus disclination (filled circle) and a minus disclination (filled square) form a defect dipole on one of the two zero-curvature circles of the torus. They are then pulled apart in the direction of the maximum curvature line (plus) and the minimum curvature line (minus), respectively.

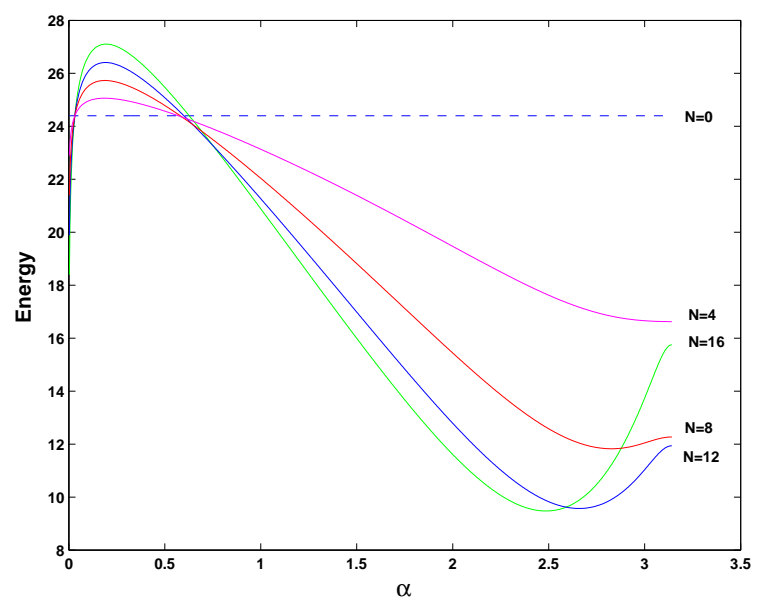

FIG. 14: Plot of the energy for the path described by Fig. 13 The disclination core energy is taken to be $0.1 K_{A}$. The aspect ratio is $r=\sqrt{5 / 4}$ and $R_{2} / a=50$. The optimal number of defect pairs in the final "buckled ring" configuration is $N^{*} / 2=7$ for this $M \approx 127,000$ particle configuration.

\section{Analytical argument for defects in the ground state for aspect ratio $r$ near 1}

Let us consider a $+/-$ disclination dipole on a zero curvature circle of the torus. Imagine slowly pulling the dipole apart until the individual $+(-)$ disclinations reach the outer(+)/inner(-) rim of the torus respectively. The total energy in this configuration is dominated by the attraction of each defect to the corresponding region of same sign curvature since the defects are too far apart for the defect-defect interaction to be important. The total 


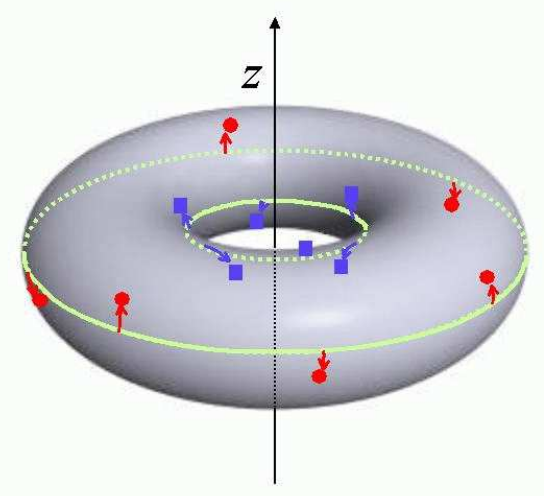

FIG. 15: The typical ground state configuration for parameters that favor defect proliferation. The arrows indicate the displacement of the equilibrium defect position from the maximal curvature circles.

energy following from Eq.(30) is therefore

$$
\mathcal{E} \approx \mathcal{E}^{d-c}=-\frac{\pi}{3} K_{A} \ln \left(\frac{r+1}{r-1}\right)
$$

where we have set $\varphi_{1}=0$ and $\varphi_{2}=\pi$ in Eq.(32). Upon approximating the defect-pair energy by its flat space value

$$
\mathcal{E}_{d d} \approx \frac{\pi}{18} K_{A} \ln \left(\frac{R_{2}}{a_{0}}\right)
$$

we find a total energy

$$
\mathcal{E}=-\frac{\pi}{3} K_{A} \ln \left(\frac{r+1}{r-1}\right)+\frac{\pi}{18} K_{A} \ln \left(\frac{R_{2}}{a_{0}}\right)+2 E_{c} .
$$

If we assume, consistent with our numerical evaluation of $\mathcal{Q}$, that the constant correction to Eq. (45) is negligible, then $E_{c}$ can be interpreted as a microscopic disclination core energy. Eq. (46) changes sign for

$$
\frac{R_{2}^{c}}{a_{0}}=\exp \left\{\frac{-36 E_{c}}{\pi K_{A}}\right\}\left(\frac{r+1}{r-1}\right)^{6} .
$$

Using $M=\frac{8 \pi^{2}}{\sqrt{3}} r\left(R_{2} / a_{0}\right)^{2}$, we conclude that defects are favored for

$$
M \lesssim M_{c}=\frac{8 \pi^{2}}{\sqrt{3}} \exp \left\{\frac{-72 E_{c}}{\pi K_{A}}\right\} r\left(\frac{r+1}{r-1}\right)^{12} .
$$

For the representative value $E_{c}=0.1 K_{A}$, we therefore find

$$
M_{c} \approx 4.6 r\left(\frac{r+1}{r-1}\right)^{12} .
$$

A comparison with our numerical results for $M_{c}$ for both vanishing core energy and $E_{c}=0.1 K_{A}$ is shown in Fig 12
- the agreement is excellent. This result also establishes that excess defects are present in the ground state for any fixed particle number provided the torus is sufficiently fat. Defects could thus be an important feature of hexatic textures for realistic vesicle sizes.

It is interesting to generalize these formulae to $p$-fold symmetric order parameters 11] on the surface of a torus. Here, hexatic order corresponds to $p=6$, nematic order to $p=2$ and tilt order to $p=1$. A hypothetical "tetradic phase" with a four-fold liquid crystalline symmetry 25] would correspond to $p=4$. The generalization of Eq.(46) for a minimally charged defect-antidefect pair with $p$-fold symmetry reads

$$
\mathcal{E}=-\frac{2 \pi}{p} K_{A} \ln \left(\frac{r+1}{r-1}\right)+\frac{2 \pi}{p^{2}} K_{A} \ln \left(\frac{R_{2}}{a_{0}}\right)+2 E_{c} .
$$

This yields a critical particle number

$$
M_{c}=\frac{8 \pi^{2}}{\sqrt{3}} \exp \left\{\frac{-2 p^{2} E_{c}}{\pi K_{A}}\right\} r\left(\frac{r+1}{r-1}\right)^{2 p} .
$$

The critical number of particles above which defects no longer appear in the ground state is therefore lower for coatings of the torus by textures of lower symmetry. Since $R_{1}$ must exceed $R_{2}$ by an amount of order $a_{0}$ for a physical torus (see Fig. (1), $(r-1)_{\min }=\left(a_{0} / R_{2}\right)$. Hence, $M_{c}$ diverges like $\left(R_{2} / a_{0}\right)^{2 p}$ in the limit of an extremely fat torus. Upon noting that $M \sim\left(R_{2} / a_{0}\right)^{2}$, we see that typically $M \ll M_{c}$ whenever $R_{2} / a_{0} \gg 1$. Thus, defects are an inevitable part of the ground state for sufficiently fat torii in all cases, except possibly for $p=1$.

\section{TEMPERATURE AND SHAPE FLUCTUATIONS}

\section{A. Connection with Two Dimensional Melting}

The renormalized Frank constant for a film in the hexatic phase has the temperature dependence 25],

$$
\frac{K_{A}(T)}{k_{B} T} \sim \frac{\xi_{+}^{2}(T)}{a_{0}^{2}},
$$

where $\xi_{+}$is the correlation length. The correlation length itself behaves in the neighborhood of the hexatic to fluid transition temperature $T_{l}$ like

$$
\xi_{+} \sim \exp \left\{\frac{b}{\sqrt{\left|T-T_{l}\right|^{1 / 2}}}\right\} .
$$

The bending rigidity has been shown to have a much weaker temperature dependence [27, 28]. Near the hexatic-fluid transition, therefore, the ratio of $K_{A} / \kappa$ diverges, which should produce larger values of $r$.

For toroidal vesicles these results change in two important ways: both the finite size and the Gaussian curvature of the torus must be taken into account. The finite area of the torus limits the growth of the correlation 
length, viz.

$$
\xi \lesssim \pi R_{2}
$$

or equivalently,

$$
\frac{K_{A}(T)}{k_{B} T} \lesssim\left(\frac{\pi R_{2}}{a_{0}}\right)^{2}
$$

so that the Frank constant no longer diverges. It is possible that the effects of Gaussian curvature will even limit $K_{A} / \kappa$ to smaller values. As discussed in the Introduction, it may be possible to "freeze-in" an aspect ratio $r \approx \sqrt{2}$ by using lipid bilayers with only short range order as a toroidal template.

\section{B. Fluctuating Hexatic Membranes}

In 29] the properties of a fluctuating hexatic membrane were investigated. It was found that the longdistance behavior is governed by a new fixed point, characteristic of a crinkled phase intermediate between a crumpled and a rigid phase. Within a large $d$ expansion, the new fixed point has the property

$$
\frac{K_{A}}{\kappa}=\frac{4 d}{3} \Rightarrow \frac{K_{A}}{\kappa}=4 \text { at } d=3 .
$$

It can be shown that for the value of $r$ corresponding to this ratio of elastic constants, additional defects should be present. The aspect ratio as a function of the elastic constants for a defect free configuration gives (see Eq.(43))

$$
r \sim \sqrt{2}
$$

a Clifford torus, which we have shown contains additional defects in the ground state.

\section{ACKNOWLEDGMENTS}

A.T. wishes to thank S. Katz for informative discussions. D.R.N. acknowledges conversations with V. Vitelli. The work of M.B. was supported by the National Science Foundation under Contract DMR-0219292 and by the U.S. Department of Energy under Contract No. DE FG02 85ER40237. The work of DRN was supported by the National Science Foundation under Grant No. DMR0231631 and by the Harvard Material Research Science and Engineering Laboratory through Grant No. DMR0213805. The work of A.T. was supported by Iowa State start-up funds.

\section{APPENDIX}

In the angular coordinates $\{\theta, \alpha\}$ of Fig. 1 (with $0 \leq$ $\theta \leq 2 \pi, 0 \leq \alpha \leq 2 \pi)$ the parametrization

$$
\begin{aligned}
& x=\left(R_{1}+R_{2} \cos \alpha\right) \cos \theta, \\
& y=\left(R_{1}+R_{2} \cos \alpha\right) \sin \theta, \\
& z=R_{2} \sin \alpha
\end{aligned}
$$

defines a torus as the locus of points $(x, y, z)$ that satisfy $\left(\sqrt{x^{2}+y^{2}}-R_{1}\right)^{2}+z^{2}=R_{2}{ }^{2}$. The dimensionless aspect ratio $r$

$$
r \equiv \frac{R_{1}}{R_{2}}
$$

is constrained to be greater than one for torii which do not self-intersect. The metric is given by

$$
d s^{2}=R_{2}^{2}\left\{(r+\cos \alpha)^{2} d \theta^{2}+d \alpha^{2}\right\} \text {. }
$$

Upon introducing a new angle variable $\varphi(0 \leq \varphi \leq 2 \pi)$ via

$$
\cos \alpha=\frac{r \cos \varphi-1}{r-\cos \varphi}
$$

Eq.(58) becomes

$$
\begin{aligned}
& x=\frac{a \sinh \rho \cos \theta}{\cosh \rho-\cos \varphi}, \\
& y=\frac{a \sinh \rho \sin \theta}{\cosh \rho-\cos \varphi}, \\
& z=\frac{a \sin \varphi}{\cosh \rho-\cos \varphi},
\end{aligned}
$$

where $a$ and $\rho$ are defined by $R_{2}=a / \sinh \rho$ and

$$
r=\cosh \rho\left(\rho=\ln \left\{r+\sqrt{r^{2}-1}\right\}\right)
$$

In these coordinates, the metric Eq.(60) becomes

$$
d s^{2}=R_{2}^{2}\left(\frac{r^{2}-1}{r-\cos \varphi}\right)^{2}\left(d \theta^{2}+\frac{d \varphi^{2}}{r^{2}-1}\right)
$$

The metric is now conformally flat, i. e. up to a $\varphi$ dependent multiplicative prefactor this is the metric of a plane with (rectangular) periodic boundary conditions. 
[2] X. Michalet and D. Bensimon, J. Phys. France II5, 263 (1995).

[3] G. Smith, E. Sirota, C. Safinya, and N. Clark, Phys. Rev. Lett. 60, 813 (1988).

[4] G.S. Smith et. al., J. Chem. Phys. 92, 4519 (1990).

[5] D. P. Siegel, Biophys. J. 76, 291 (1999).

[6] V. Markin and J. Albanes, Biophys. J. 82, 693 (2002).

[7] Y. Kozlovsky and M. Kozlov, Biophys. J. 82, 882 (2002).

[8] W. Helfrich, Z. Naturforsch 28C, 693 (1973).

[9] F. Julicher, U. Seifert, and R. Lipowsky, J. Phys. France II3, 1681 (1993).

[10] U. Seifert, Adv. Phys. 46, 13 (1997).

[11] T. C. Lubensky and J. Prost, J. Phys. France II2, 371 (1992); see also R. M. L. Evans, J. Phys. France II5, 507 (1995) arXiv:cond-mat/9410010.

[12] A. D. Dinsmore, M. F. Hsu, M. G. Nikolaides, M. Marquez, A. R. Bausch and D. A. Weitz, Science 298, 1006 (2002).

[13] C. M. Murray, in Bond-Orientational Order in Condensed Matter Systems, edited by K. Strandburg (Springer-Verlag, Berlin, 1992).

[14] A. R. Bausch, M. J. Bowick, A. Cacciuto, A. D. Dinsmore, M. F. Hsu, D. R,. Nelson, M. G. Nikolaides, A. Travesset and D. A. Weitz, Science 299, 1716 (2003) arXiv:cond-mat/0303289.

[15] P. G. deGennes and J. Prost, The Physics of Liquid Crystals (Clarendon Press, Oxford, 1993). For tilt order on a torus, we expect a maximum of two positive disclinations on the outer wall, with compensating negative defects on the inner wall. For nematic order, the maximum number of + or - defects is 4 .

[16] V. Vitelli and D. R. Nelson, to be published.

[17] J. Selinger and D. R. Nelson, Phys. Rev. A39, 3135 (1989).

[18] F. David, in Statistical Mechanics of Membranes and Surfaces, edited by D. Nelson, T. Piran and S. Weinberg (World Scientific, Singapore, 1989); see also Ref. 20].

[19] M. Bowick, D.R. Nelson, and A. Travesset, Phys. Rev. B62, 8738 (2000) arXiv:cond-mat/9911379.

[20] M. Bowick and A. Travesset, J. Phys. A: Math. Gen. 34, 1525 (2001) arXiv:cond-mat/0005356.

[21] See, e.g., S.B. Morse and H. Feshbach, Methods of Theoretical Physics (Addison Wesley, New York, 1967) Vol. 1, p. 666 and Vol. 2, p.1301.

[22] J. Polchinski, String Theory, Vol. I (Cambridge University Press, Cambridge, 1998).

[23] D. Mumford, Tata Lectures on Theta I (Birkhaüser, Boston, 1983).

[24] E.T. Whittaker and G.N. Watson, A Course of Modern Analysis, Fourth Edition (Cambridge University Press, Cambridge, 1927).

[25] D.R. Nelson and B.I. Halperin, Phys. Rev. B19, 2457 (1979).

[26] A. P. Young, Phys. Rev. B19, 1855 (1979).

[27] H. Kleinert, Phys. Lett. A114, 263 (1986).

[28] D. Forster, Phys. Lett. A114, 115 (1986).

[29] F. David, E. Guitter, and L. Peliti, J. de Physique 48, 1085 (1987). 